\title{
BMJ Open Exercise duRing Active Surveillance for prostatE cancer - the ERASE trial: a study protocol of a phase II randomised controlled trial
}

\author{
Dong-Woo Kang, ${ }^{1}$ Adrian S Fairey, ${ }^{2}$ Normand G Boulé, ${ }^{1}$ Catherine J Field, ${ }^{3}$ \\ Kerry S Courneya ${ }^{1}$
}

To cite: Kang D-W, Fairey AS, Boulé NG, et al. Exercise duRing Active Surveillance for prostatE cancer-the ERASE trial: a study protocol of a phase II randomised controlled trial. BMJ Open 2019;9:e026438. doi:10.1136/ bmjopen-2018-026438

- Prepublication history and additional material for this paper are available online. To view these files, please visit the journal online (http://dx.doi org/10.1136/bmjopen-2018026438).

Received 1 September 2018 Revised 28 February 2019 Accepted 4 March 2019
Check for updates

C Author(s) (or their employer(s)) 2019. Re-use permitted under CC BY-NC. No commercial re-use. See rights and permissions. Published by BMJ.

${ }^{1}$ Faculty of Kinesiology, Sport, and Recreation, University of Alberta, Edmonton, AB, Canada

${ }^{2}$ Division of Urology, Department of Surgery, Facultyof Medicine \& Dentistry, University of Alberta,

Edmonton, $A B$, Canada

${ }^{3}$ Department of Agricultural, Food and Nutritional Science, University of Alberta, Edmonton, $A B$, Canada

Correspondence to

Kerry S Courneya;

kerry.courneya@ualberta.ca

\section{ABSTRACT}

Introduction Active surveillance (AS) is the preferred primary treatment strategy for men with low-risk clinically localised prostate cancer (PCa); however, the majority of these men still receive radical treatment within 10 years due to disease progression and/or fear of cancer progression. Interventions designed to suppress tumour growth, mitigate fear of cancer progression and precondition men for impending radical treatments are an unmet clinical need. Exercise has been shown to delay the progression of prostate tumours in animal models, improve physical and functional health and manage psychological outcomes in cancer patients; however, these outcomes have not been demonstrated in PCa patients undergoing AS.

Methods and analysis This phase II randomised controlled trial will randomise 66 men undergoing AS to either an exercise group or a usual care group. The exercise group will perform a 12-week, supervised, high-intensity interval training programme, consisting of 3 sessions/week for $28-40 \mathrm{~min} /$ session. The primary outcome will be cardiorespiratory fitness. Secondary outcomes will include immunosurveillance and cancerrelated biomarkers, psychosocial outcomes including fear of cancer progression and quality of life and physical function. Exploratory outcomes will include clinical indicators of disease progression. The trial has $80 \%$ power to detect a significant between-group difference in $\mathrm{VO}_{2 \text { peak }}$ of $3.5 \mathrm{~mL} / \mathrm{kg} / \mathrm{min}$ with a two-tailed alpha level $<0.05$ and a $10 \%$ dropout rate.

Ethics and dissemination The study has received full ethical approval from the Health Research Ethics Board of Alberta - Cancer Committee (Protocol Number: HREBA.CC17-0248). The findings of the study will be disseminated through public and scientific channels.

Trial registration number NCT03203460; Pre-results.

\section{INTRODUCTION}

Conventionally, prostate cancer (PCa) is treated immediately by radical prostatectomy, radiation therapy and/or hormonal therapy. ${ }^{1}$ These treatments improve overall survival; however, they are expensive and cause side-effects such as sexual and urinary dysfunction,

\section{Strengths and limitations of this study}

- This study will be the first randomised controlled trial to test a high-intensity interval training intervention in men with prostate cancer undergoing active surveillance.

- The intervention will be supervised and designed to maximise improvements in aerobic fitness and biological outcomes.

- This study will examine important intermediate outcomes including biomarkers related to cancer surveillance and tumour growth and fear of cancer progression.

- This study is not powered to examine the key clinical outcomes of progression to radical treatment or survival.

- The intervention is only 12 weeks long and there is a usual care crossover at postintervention that will confound longer term comparisons.

fatigue, loss of lean body mass, muscle weakness and reduced quality of life. ${ }^{2-4}$ Active surveillance (AS) has emerged as an alternative clinical practice that is offered to men with low-to-favourable intermediate risk of PCa. ${ }^{5}$ In AS, men with PCa do not receive any immediate radical treatments but are closely monitored for any signs of tumour progression. The obvious advantage of AS is that it allows early-stage PCa patients to avoid treatment-related side-effects without compromising their PCa-specific and overall survival. ${ }^{36}$ Moreover, there is substantial cost saving over radical treatments. ${ }^{7-10}$

Unfortunately, many men with PCa on AS eventually experience disease progression and require radical treatments. Approximately $30 \%$ of men on AS receive radical treatments within 3 years and about $55 \%$ within 10 years. ${ }^{6}$ Moreover, even without an objective progression of their disease, many AS patients experience anxiety and fear of cancer progression based on having an untreated tumour with a 


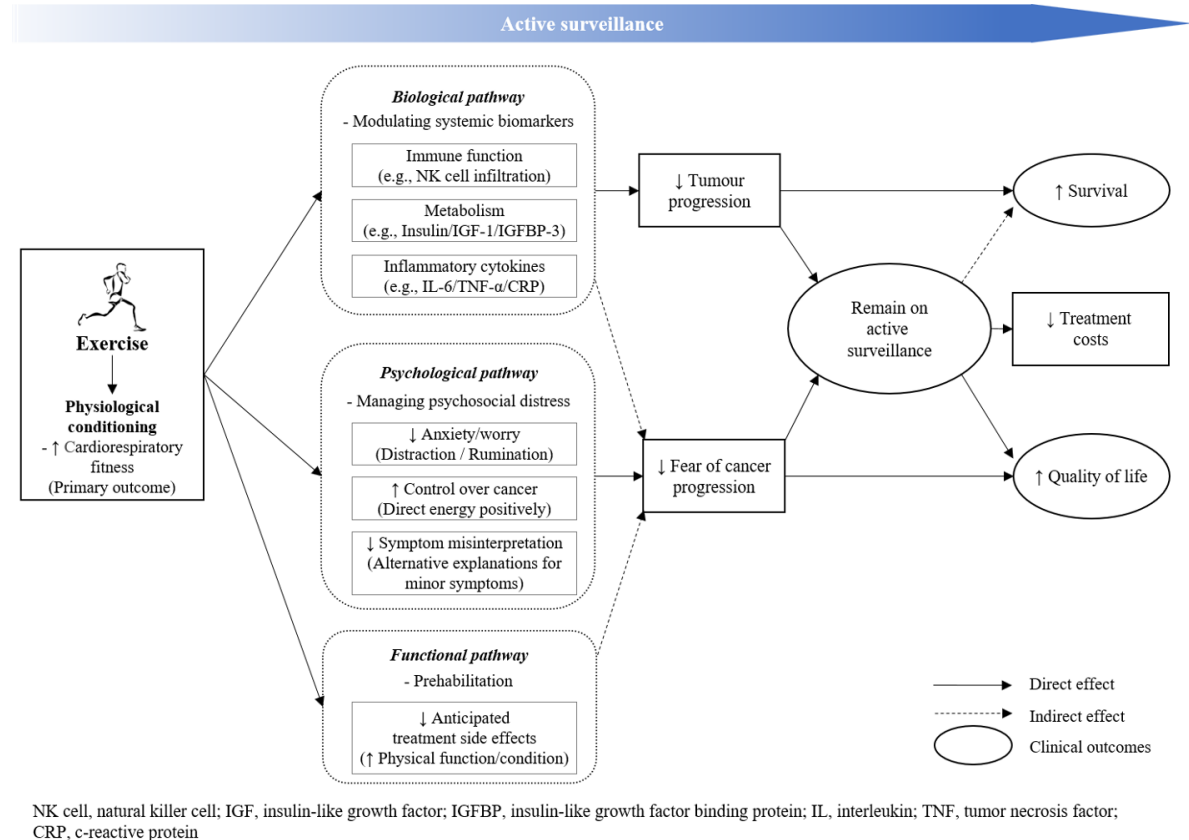

Figure 1 Proposed effects of exercise during active surveillance in prostate cancer patients.

chance of progression. ${ }^{11} 12$ Fear of cancer progression and uncertainty of illness are associated with a poor quality of life ${ }^{13}$ which may prompt these men and their doctors to opt for radical treatments as a way of managing the fear and anxiety. ${ }^{14-17}$

Evidence shows that exercise may be beneficial to men with PCa on AS in three potential pathways proposed in our model (figure 1). First, exercise that is sufficient to improve cardiorespiratory fitness may be linked to slower PCa progression. ${ }^{18}$ Several possible biological mechanisms have been studied in animal ${ }^{19-21}$ and human ${ }^{22}$ models, including improved immune activity through aerobic exercise which may play a critical role $;{ }^{23}$ however, these findings have not been confirmed in men with PCa. Second, exercise has been shown to improve health-related fitness, ${ }^{24-27}$ treatment-related side-effects, ${ }^{24}{ }^{26}$ physical functioning, ${ }^{25-30}$ body composition, ${ }^{25-28}$ fatigue ${ }^{26-28} 30$ and quality of life $e^{26-283031}$ during and after radical PCa treatments, but few studies to date have focused on the AS setting or examined fear of cancer progression. Last, given the eventuality of receiving radical treatment for many PCa patients on AS, ${ }^{6}$ exercise may help these men improve overall physical condition/function prior to impending radical treatments (ie, prehabilitation) ${ }^{32}$ which may reduce anticipated treatment-related side-effects. However, current studies are only preliminary and few have focused on PCa patients on AS. Therefore, we hypothesised that exercise may be a cost-effective intervention that will provide clinical benefits to men with $\mathrm{PCa}$ undergoing AS.

The primary objective of the Exercise duRing Active Surveillance for prostatE cancer (ERASE) Trial is to examine the effects of exercise on cardiorespiratory fitness in PCa patients undergoing AS. The secondary objectives are to examine the effects of exercise on: (1) biological markers linked to immune surveillance and PCa, as well as tumour-related metabolic and proinflammatory biomarkers, (2) patient-reported outcomes including fear of cancer progression, anxiety and quality of life and (3) health-related fitness outcomes including physical function and body composition. The exploratory objectives are to examine the effects of exercise on clinical indicators of cancer progression including progression to radical treatments. In this paper, the detailed study design and protocol of the ERASE trial are described based on the Standard Protocol Items for Randomised Trials (SPIRIT) guideline.

\section{METHODS AND ANALYSIS \\ Study design}

The ERASE trial will be a prospective, single centre, two-armed, phase II randomised controlled trial at the University of Alberta and the Northern Alberta Urology Centre (NAUC) in Edmonton, Alberta, Canada. The proposed study activities and assessments by timepoints based on the SPIRIT guideline are presented in table 1 , and the proposed study participant flow diagram throughout the study is shown in figure 2 .

\section{Study population}

Men will be eligible if they are: (1) $\geq 18$ years old, (2) diagnosed with very low, low or favourable intermediate grade localised PCa defined by the 2017 National Comprehensive Cancer Network (NCCN) Guidelines for PCa, ${ }^{33}$ (3) undergoing AS as the primary treatment option with no plans for radical treatment at the time of recruitment, (4) medically cleared to participate in the study as determined by their treating urologist and a certified clinical exercise physiologist using the Physical Activity Readiness 


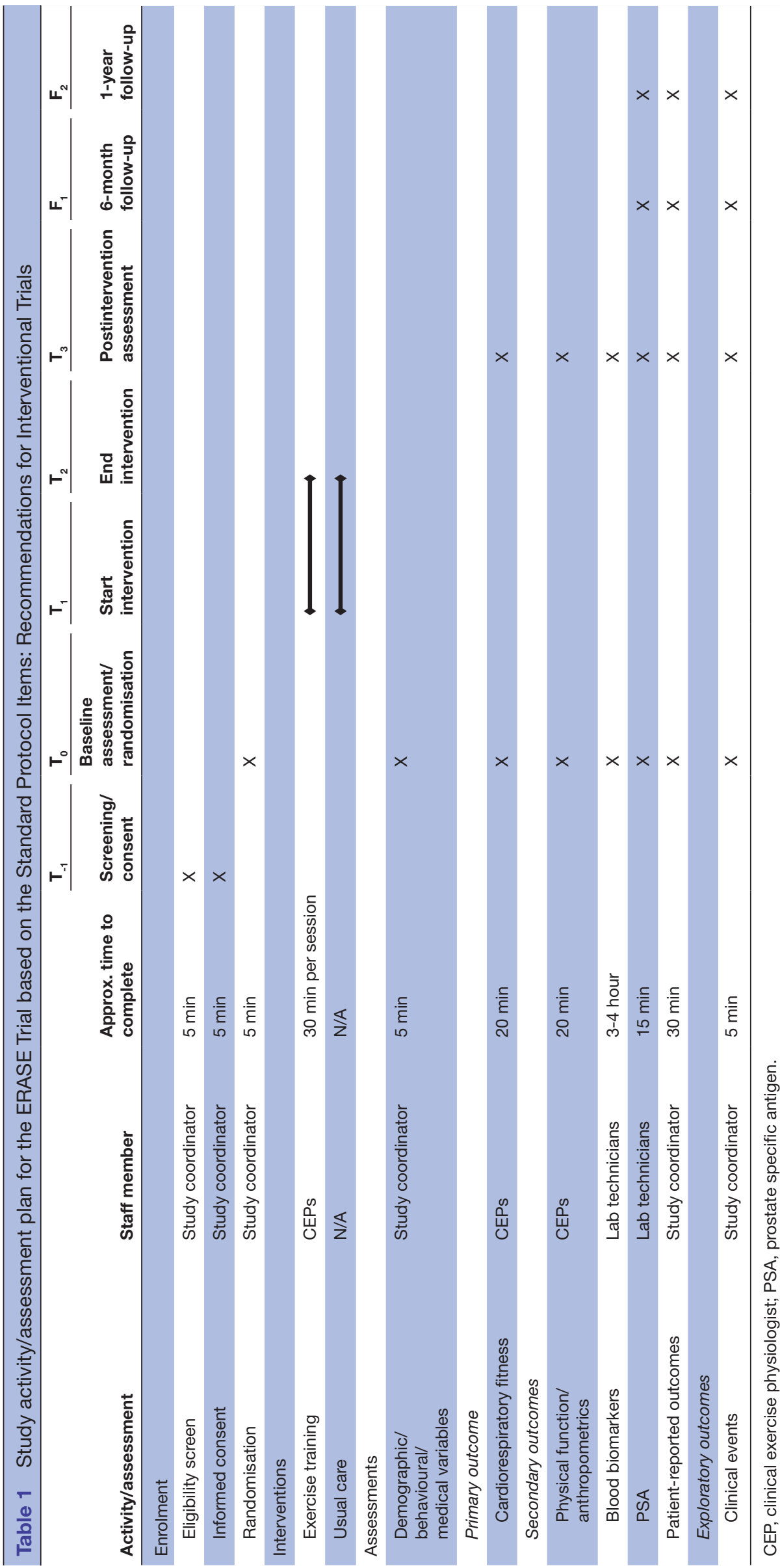

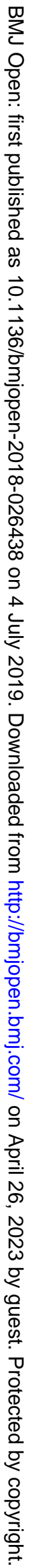




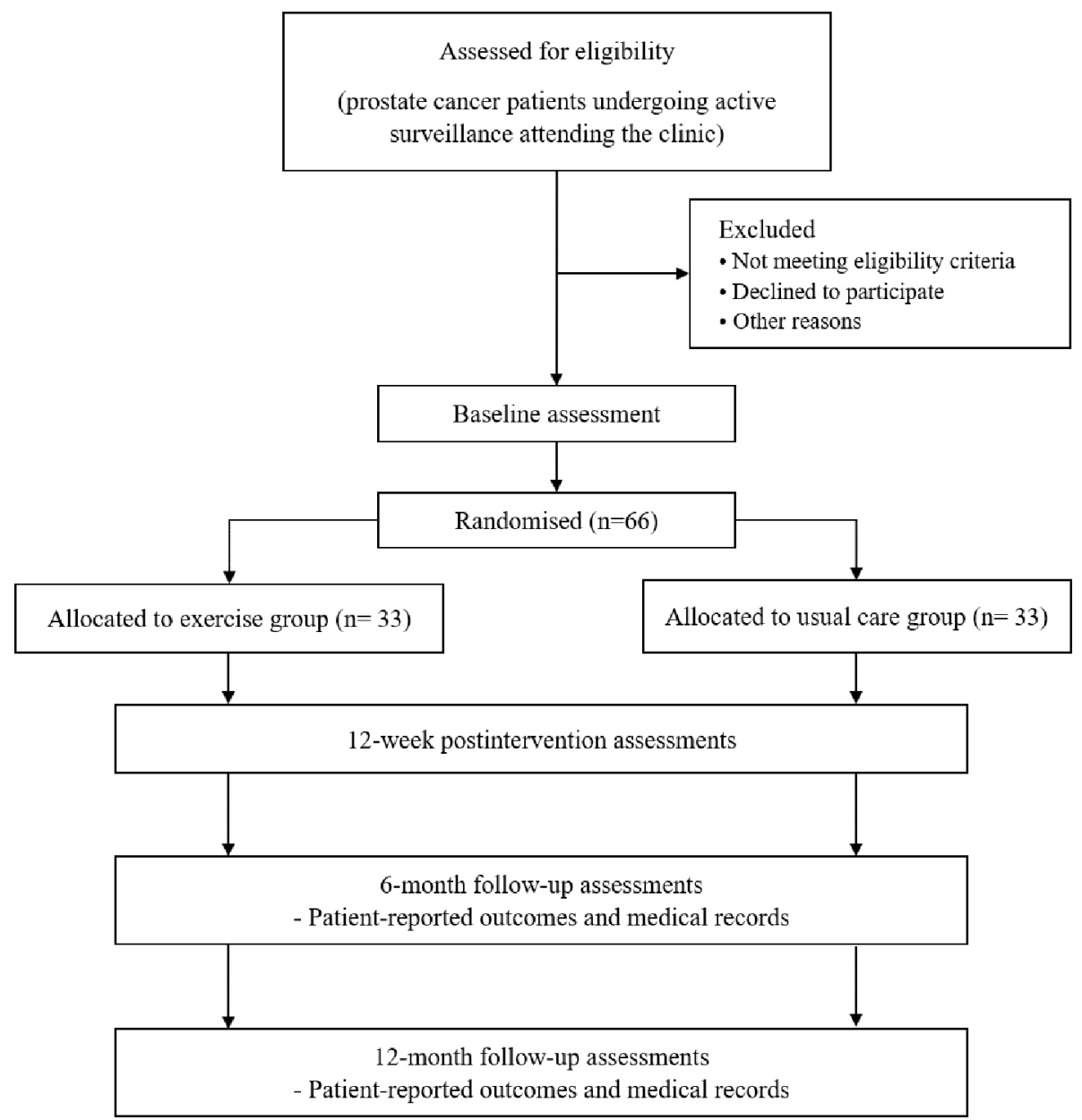

Figure 2 Proposed patient flow diagram of the ERASE Trial.

Questionnaire, ${ }^{34}$ (5) able to complete the assessment for the primary outcome of the study (ie, maximal aerobic exercise testing) at baseline, (6) free of uncontrolled medical conditions that could be exacerbated with exercise, (7) not participating in any structured high-intensity exercise defined as $0 \mathrm{~min}$ of vigorous intensity exercise during a typical week in the past month measured by the modified Godin Leisure-Time Exercise Questionnaire $(\text { GLTEQ })^{35}$ and (8) willing to perform all required study assessments and interventions and be randomised to either a 12-week supervised exercise training programme at the University of Alberta or continue with their usual activity for 12 weeks.

\section{Recruitment}

Recruitment will be conducted through the NAUC at the Kaye Edmonton Clinic, Edmonton, Canada. Patients will be screened for eligibility through the NAUC medical record and eligible patients will be briefly informed about the study by their physicians during their checkup visits. All interested patients will receive a detailed explanation of the study from the study coordinators and be further screened for current physical activity readiness and participation level for eligibility. Those patients who are eligible and agree to participate will be asked to provide written informed consent for study participation and blood banking.

\section{Randomisation and blinding}

Participants will be randomly assigned to either the exercise group or the usual care group in a 1:1 ratio on completion of baseline assessment. The allocation sequence will be produced by computer-generated block randomisation numbers and concealed from study staff involved in recruitment and baseline assessment. Due to the nature of the exercise intervention, it is not possible to blind participants or interventionists to group allocation. Outcome assessors will not always be blinded to group allocation for the physical fitness and body composition outcomes due to logistical issues, but they will be trained in standardised testing procedures. Outcome assessors for other secondary and exploratory outcomes will be blinded.

\section{Interventions}

Exercise group

Patients randomised to the exercise group will be asked to complete a 12-week, supervised high-intensity interval training (HIIT) programme (table 2; figure 3). The exercise will consist of alternating vigorous and low-intensity 
Table 2 The 12-week high-intensity interval training periodisation scheme and programme details in the ERASE Trial

\begin{tabular}{|c|c|c|c|c|c|c|c|c|c|c|c|c|}
\hline \multirow[b]{2}{*}{$\begin{array}{l}\text { Intervention } \\
\text { period }\end{array}$} & \multirow[b]{2}{*}{ Week } & \multirow[b]{2}{*}{ Warm-up } & \multicolumn{4}{|c|}{ High-intensity phase } & \multicolumn{4}{|c|}{ Recovery phase } & \multirow[b]{2}{*}{$\begin{array}{l}\text { Cool- } \\
\text { down }\end{array}$} & \multirow[b]{2}{*}{$\begin{array}{l}\text { Total } \\
\text { duration } \\
\text { (min) }\end{array}$} \\
\hline & & & $\begin{array}{l}\text { Intensity (\% } \\
\text { vo }_{2 \text { peak }} \text { ) }\end{array}$ & $\begin{array}{l}\text { Duration } \\
\text { (min) }\end{array}$ & $\begin{array}{l}\text { No. of } \\
\text { intervals }\end{array}$ & $\begin{array}{l}\text { Sum of } \\
\text { high-intensity } \\
\text { duration (min) }\end{array}$ & $\begin{array}{l}\text { Intensity } \\
\left(\% \mathrm{VO}_{2 \text { peak }}\right)\end{array}$ & $\begin{array}{l}\text { Duration } \\
\text { (min) }\end{array}$ & $\begin{array}{l}\text { No. of } \\
\text { intervals }\end{array}$ & $\begin{array}{l}\text { Sum of } \\
\text { recovery } \\
\text { duration (min) }\end{array}$ & & \\
\hline Period 1 & $1-4$ & \multirow{3}{*}{$\begin{array}{l}5 \mathrm{~min} \\
\text { at } 60 \% \\
\mathrm{VO}_{2 \text { peak }}\end{array}$} & 85 & 2 & $5-8$ & $10-16$ & 40 & 2 & $4-7$ & $8-14$ & \multirow{3}{*}{$\begin{array}{l}5 \mathrm{~min} \\
\text { at } 30 \% \\
\mathrm{VO}_{2 \text { peak }}\end{array}$} & $28-40$ \\
\hline Period 2 & $5-8$ & & 90 & 2 & 8 & 16 & 40 & 2 & 7 & 14 & & 40 \\
\hline Period 3 & $9-12$ & & 95 & 2 & 8 & 16 & 40 & 2 & 7 & 14 & & 40 \\
\hline
\end{tabular}

intervals performed on a treadmill. Exercise frequency will be 3 times per week and the duration of each session will progress from 28 to $40 \mathrm{~min}$. The intensity of the exercise will be modified by changing the treadmill speed and/or grade prescribed at specific workloads corresponding to $85 \%-95 \%$ of peak oxygen consumption $\left(\mathrm{VO}_{2 \text { peak }}\right)$ measured at baseline. The exercise programme consists of: (1) 'warm-up' for 5 min at a workload corresponding to $60 \%$ of $\mathrm{VO}_{2 \text { peak }}$, (2) 'high-intensity' phase for $2 \mathrm{~min}$ at a gradually increasing workload corresponding to $85 \%-95 \%$ of $\mathrm{VO}_{2 \text { peak }}$ (ie, $85 \%$ in the 1 st-4th week, $90 \%$ in the 5 th-8th week and $95 \%$ in the 9 th-12th week), (3) 'recovery' phase for 2 min at a workload corresponding to $40 \%$ of $\mathrm{VO}_{2 \text { peak }}$, (4) repeated sets of 'high-intensity' and 'recovery' phases (ie, increasing from 5 to 8 sets in the 1st-4th week for initial training adaptation and 8 sets in the 5th-12th week), and (5) 'cool-down' for $5 \mathrm{~min}$ at a workload corresponding to $30 \%$ of $\mathrm{VO}_{2 \text { peak }}$ ). Stretching for lower body muscles (eg, quadriceps, hamstrings and calves) will be followed after each exercise session for $5 \mathrm{~min}$. For the purpose of monitoring exercise intensity and safety, heart rates (HRs) will continuously be monitored throughout each session using a HR monitor (Polar T31; Woodbudy, NY, USA). Over the 12-week intervention period, exercise adherence to the prescribed exercise programme will be monitored and recorded based on attendance at the scheduled exercise sessions, and completion of the prescribed intensity, duration and number of intervals during each exercise session. Strategies to maximise adherence will include appointment-based sessions, flexible scheduling, individualised exercise programme, supervision by certified clinical exercise physiologists, free parking and use of the Behavioural Medicine Fitness Centre which is available only to cancer patients participating in the clinical exercise trials. Any missed sessions will trigger a telephone call with a rescheduling of the exercise session as soon as possible.

\section{Usual care group}

Currently, the standard AS care at our site does not include any formal exercise programme or advice. Consequently, participants in the usual care group will be asked not to change their exercise levels from baseline during the 12-week study period. After the postintervention assessments at 12 weeks, patients in the usual care group will be offered a 4-week HIIT programme at our facility and/or referred to a 12-week community-based program. ${ }^{36}$

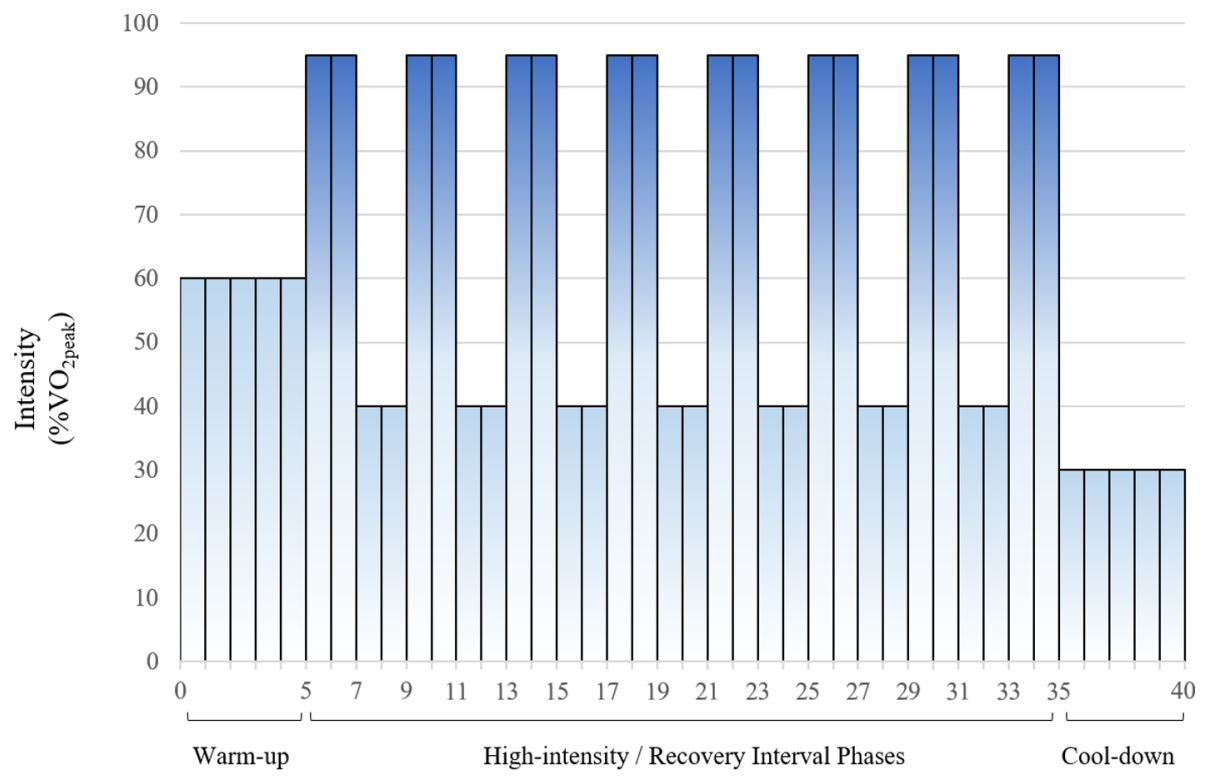

Time (Minute)

Figure 3 High-intensity interval training programme in the ERASE Trial. 


\section{Outcome measurements}

All study outcomes will be measured at baseline and postintervention at 12 weeks. Also, questionnaires for patient-reported outcomes, prostate-specific antigen (PSA) for disease progression and medical records for clinical events will be obtained at 6-month and 1-year follow-ups. Study outcome assessment timelines are summarised in table 1 and the validity and reliability of each outcome are described in the online supplementary table.

\section{Primary outcome}

The primary outcome of the study will be cardiorespiratory fitness. Cardiorespiratory fitness will be measured as $\mathrm{VO}_{2 \text { peak }}$ by clinical exercise physiologists who are trained for the standardised treadmill testing protocol (modified Bruce protocol). ${ }^{37}$ The test will be conducted on a treadmill (Woodway 4Front; Waukesha, WI, USA) with direct measures of cardiorespiratory variables using a metabolic cart (Parvo Medics TrueOne 2400; Sandy, UT, USA). After a 5-min warm-up, the test will begin at $1.7 \mathrm{mph}$ and $0 \%$ grade, and the speed and incline will increase every $3 \mathrm{~min}$ until volitional exhaustion or any testing contraindications occur. During the test, oxygen uptake and HR will be recorded continuously, and blood pressure (BP) using a manual sphygmomanometer, oxygen saturation using a pulse oximeter and rated perceived exertion level using the modified Borg Scale ${ }^{38}$ will be measured every 3 min. After the test, a 5 -min active recovery will be conducted at $1.7 \mathrm{mph}$ and $0 \%$ grade, and $\mathrm{HR}$ and $\mathrm{BP}$ will be measured at 2 and 5 min during recovery. $\mathrm{VO}_{2 \text { peak }}$ is defined as the highest oxygen uptake value recorded during the test expressed relative to body mass (ie, $\mathrm{mL} / \mathrm{kg}^{-1} \cdot \mathrm{min}^{-1}$ ).

\section{Secondary outcomes}

Blood-based outcomes will include biomarkers related to immune surveillance (eg, natural killer (NK) cell counts and cytotoxic activity), PCa progression (eg, PSA), inflammatory cytokines (eg, interferon- $\gamma$, interleukin (IL) $-1 \beta$, IL-2, IL-4, IL-6, IL-8, IL-10, IL-12p70, IL-13, tumour-necrosis factor (TNF)- $\alpha$ and c-reactive protein (CRP)), metabolism (eg, serum insulin, glucose, HbAlc, insulin-like growth factor (IGF)-1, IGF binding protein-3, adiponectin and leptin), lipid profiles (eg, high- and low-density lipoprotein cholesterol and triglycerides) and sex hormones (eg, testosterone and progesterone). Blood samples will be collected after 12 hours of fasting at the Kaye Edmonton Clinic Laboratory Services and then directly sent to the biochemistry lab in the Li Ka Shing Centre for Health Research Innovation at the University of Alberta. On collection, mononuclear cells on fresh blood will be isolated on a ficoll gradient and characterised using specific monoclonal antibodies and flow cytometry. The plasma will be frozen and stored in a $-80^{\circ} \mathrm{C}$ freezer, monitored and alarmed and only accessible to authorised personnel. For markers that will be assayed on frozen blood, the samples from baseline and postintervention for each subject will be assayed at the same time for more accurate assessment of change using paired assays plated in random order. The immune phenotype identification and biological measures will be performed by trained technicians who are blinded to the treatments and trained in the standardised protocol. Appropriate standard and reference samples will be included in each assay.

Patient-reported outcomes will include fear of cancer progression using the Fear of Cancer Recurrence Inventory $^{39}$ and Cancer Worry Scale ${ }^{40}$, health-related quality of life using the European Organization for Research and Treatment of Cancer- Quality of Life Questionnaire-C30 ${ }^{41}$ and PCa-specific health-related quality of life and symptoms using the Expanded Prostate Cancer Index Composite-26. General and prostate-specific anxiety will be assessed using the Spielberger State-Trait Anxiety Inventory $^{42}$ and the Memorial Anxiety Scale for Prostate Cancer, ${ }^{43}$ respectively. Depression will be measured using the Center for Epidemiologic Studies Depression Scale, ${ }^{44}$ fatigue using the Functional Assessment of Cancer Therapy- Fatigue, ${ }^{45}$ perceived stress using the Perceived Stress Scale, ${ }^{46}$ self-esteem using the Rosenberg Self-Esteem Scale $^{47}$ and motivation to exercise using Theory of Planned Behaviour. ${ }^{48}$ Exercise levels will be assessed using the modified GLTEQ. ${ }^{35}$ Patient-reported outcomes will be collected using a set of questionnaires which will be sent to patients via mail and patients will be asked to complete within a week from each assessment date. Physical function will be assessed using the Senior's Fitness Test ${ }^{49}$ which includes tests for lower body strength (chair stand), upper body strength (arm curl), lower body flexibility (chair sit and reach), upper body flexibility (back scratch), agility (8-foot up and go) and aerobic fitness (6-min walk test). Body composition will include weight, height, and waist and hip circumferences using scales and tape measures. Physical function and body composition assessments will be conducted by clinical exercise physiologists following the standardised protocols. ${ }^{49} 50$

\section{Exploratory outcomes}

Exploratory outcomes will include progression to invasive PCa treatment and any clinically relevant events. Clinical events will be reviewed by research staff who are authorised to have access to patient medical records.

\section{Demographic, behavioural and medical variables, and adverse} events

Demographic and behavioural information will be obtained using a self-reported questionnaire at baseline. The questionnaire will assess age, ethnicity, education, marital status, income, employment status, smoking and alcohol consumption. Patients' medical information will be abstracted from the NAUC electronic medical record at baseline, including tumour-related information and any complications or comorbidities. Adverse events will be monitored, assessed and recorded during exercise testing and before and after each exercise session throughout the intervention period. 


\section{Sample size}

A sample size of 60 participants (30 per group) provides $80 \%$ power using a two-tailed alpha $<0.05$ to detect a clinically meaningful between-group difference of 1-metabolic equivalent task (1-MET; $3.5 \mathrm{~mL} / \mathrm{kg} / \mathrm{min}$ ) on our primary outcome of $\mathrm{VO}_{2 \text { peak }}$, assuming a $\mathrm{SD}$ of $5.6 \mathrm{~mL} / \mathrm{kg} / \mathrm{min}$ and adjustment for baseline value and other prognostic covariates. It will also be sufficient for detecting differences in our secondary biomarkers and patient-reported outcomes. ${ }^{51}$ Considering a potential dropout rate of $<10 \%$ based on our previous exercise oncology trials, ${ }^{52-55}$ a total of 66 participants (33 per group) will be randomised. This power will also be sufficient for detecting differences in our secondary biomarkers and patient-reported outcomes if the effects are moderate (ie, a standardised effect size of $d \geq 0.6$ approximately). This power is not sufficient for detecting potentially meaningful differences in any of the exploratory clinical outcomes. Given that the purpose of this phase II trial is to inform larger phase II and III trials, the patient-reported and clinical outcomes will also be interpreted for potential clinical significance based on the direction and magnitude of numerical differences.

\section{Data collection and management}

All data will be collected and stored anonymised in the Behavioural Medicine Laboratory at the University of Alberta in a locked filing cabinet or in a secured, web-based application for electronic data using the Research Electronic Data Capture tools. ${ }^{56}$ Data files will only be accessible to study investigators and authorised personnel for data entry and quality control purposes. Data quality will be assured using the double data entry/data comparison and data quality modules ${ }^{56}$ by an independent research personnel who will be blinded to the origin of the data.

\section{Statistical analysis}

We will conduct descriptive analyses for participant characteristics, intervention adherence and compliance rates, adverse events, and exploratory outcomes of disease progression and clinical events. Analyses of covariance will be performed for the primary and secondary outcomes to compare the between-group differences at postintervention after adjustment for potential covariates. Covariates will be selected a priori and include baseline value and other baseline variables that appear unbalanced between the two groups. If an outcome variable presents a non-normal distribution, $\log$ data transformations will be conducted for analyses. All statistical analyses will include all study participants with baseline and follow-up data and will be conducted based on the intention-to-treat principle. Any reasons for missing intervention sessions or study drop-out will be assessed and reported. If missing data is $<10 \%$, we will conduct a complete case analysis. If missing data is $\geq 10 \%$, we will employ a multiple imputation missing data strategy. ${ }^{57}$ Interpretation of the data will be based on the p-value of a two-tailed alpha $<0.05$, meaningful effect sizes ( $d \geq 0.5$ for the secondary outcomes) and the general patterns of the findings.

\section{Patient and public involvement}

The conception, design, and outcome measures for this study were informed by previous research in PCa patients concerning their priorities for important outcomes and their preferences for exercise intervention. Patients will not be directly involved in the recruitment or conduct of the study. The burden of intervention will be assessed by patients themselves at postintervention through a questionnaire (eg, 'Exercise programme too demanding or difficult'). The results and current progress of the study will be disseminated to the study participants via email and a study webpage (www.erasestudy.com).

\section{DISCUSSION}

AS has become a preferred strategy for the management of early-stage PCa patients; however, many men experience progression and anxiety. Cost-effective interventions that can slow tumour progression, manage fear of cancer progression and prepare PCa patients on AS for impending radical treatments would be highly beneficial. To date, however, no such interventions are offered as standard of care in this clinical setting.

There have been a few lifestyle intervention trials in men with PCa on AS. One randomised controlled trial with 93 PCa patients on AS provided a 1-year lifestyle programme comprising a vegan diet, walking exercise and stress management. ${ }^{58-62}$ At the 3-month interim analyses, the study reported significant improvements in cardiovascular risk factors such as body mass index, BP and lipid profile, as well as several indicators of psychological functioning including mental component summary, intrusive thoughts and avoidance. ${ }^{61}$ PSA levels as a cancer progression outcome were not significantly changed but clinically meaningful changes were found. ${ }^{61}$ At 1-year postintervention, there were similar improvements as at 3 months, and total PSA levels were significantly reduced by $4 \%$ in the intervention group while there was a $6 \%$ increase in the control group. ${ }^{58}$ Finally, overall health-related quality of life increased in the intervention group compared with the control group. ${ }^{60} \mathrm{~A}$ follow-up for clinical events at 2 years noted that 2 of $43(5 \%)$ patients in the intervention group compared with 13 of 49 (27\%) patients in the control group had proceeded to radical PCa treatments. ${ }^{61}$ This study provided promising evidence that an intensive lifestyle intervention in PCa patients on AS may slow the progression of the disease, improve some aspects of quality of life and decrease the rate of radical treatments; however, it is unclear whether the benefits were due to diet, exercise or stress management.

Another randomised controlled trial in 26 men with PCa undergoing AS tested the effects of a 6-month combined whole-grain diet and vigorous exercise programme. ${ }^{63}$ The exercise programme included non-supervised aerobic exercise 3 times/week of $45 \mathrm{~min} /$ session targeting $70 \%$ of maximal HR, in addition to at least 10000 steps daily. The combined intervention resulted in a significant improvement in $\mathrm{VO}_{2 \text { peak }}$ compared with the control group but no 
significant differences in body composition, cardiometabolic outcomes or PSA levels. Moreover, several cohort, cross-sectional and systematic review studies have also suggested the potential roles of lifestyle characteristics for cancer-related outcomes ${ }^{63-66}$ however, the isolated effects of exercise during AS on physical fitness, fear of cancer progression or PCa-related outcomes were not investigated.

More recently, one completed and one ongoing trial, focused on exercise in PCa patients on AS, have been reported. Bourke et al conducted an exercise clinical trial on $50 \mathrm{PCa}$ patients undergoing $\mathrm{AS}^{67}$ The exercise programme included supervised exercise training sessions with behavioural support, targeting $150 \mathrm{~min}$ of moderate to vigorous exercise per week over 12 months. Although preliminary, the findings suggest exercise is a feasible and acceptable intervention during AS. Also, Galvão and colleagues have reported the protocol for a 3-year long randomised trial examining the effects of a long-term aerobic and resistance exercise programme on the need for invasive treatment. ${ }^{68}$ Compared with these two studies, the ERASE trial will address the efficacy of a relatively shorter term (ie, 12 weeks) high-intensity aerobic exercise on various clinically-relevant outcomes including tumour-related biomarkers, fear of cancer progression and disease progression outcomes.

Our primary outcome of cardiorespiratory fitness was selected for the following reasons. First, evidence shows that improvement in cardiorespiratory fitness is inversely correlated with prostate-specific antigen doubling time $\left(\mathrm{R}^{2}=0.41, \mathrm{p}<0.003\right)$, which is one of the indicators of biochemical and clinical progression of PCa. ${ }^{18}$ These data suggest that improvement in cardiorespiratory fitness through aerobic training induces systemic and physiological changes that may interact with molecular suppression of cancer progression. ${ }^{18}$ It is also supported by epidemiological studies showing inverse association between cardiorespiratory fitness and development of PCa. ${ }^{69}{ }^{70} \mathrm{In}$ addition, cardiorespiratory fitness has important clinical implications for cardiovascular and overall prognosis of PCa. ${ }^{69} \mathrm{PCa}$ patients are at a higher risk of cardiovascular morbidity ${ }^{7172}$ and mortality ${ }^{72}$ and are three times more likely to die of cardiovascular disease than of PCa. ${ }^{6}$ Cardiorespiratory fitness is an established surrogate marker for cardiovascular disease and overall survival ${ }^{74} 75$ and impaired cardiopulmonary fitness, commonly reported among cancer patients, is correlated with cancer-related symptoms and clinical outcomes. ${ }^{76}$ Thus, maintaining or improving cardiopulmonary fitness is of clinical importance in PCa patients on $\mathrm{AS}^{76}$

We elected to test a HIIT exercise intervention for several reasons. In terms of maximising the potential benefits of exercise in AS patients, a body of evidence supports the thesis that higher-intensity exercise programmes often produce greater health benefits compared with moderate-intensity continuous training. ${ }^{77-81}$ HIIT aerobic exercise is a type of high-intensity exercise, alternating short periods of intense exercise and active recovery. HIIT has been shown to induce greater physical and physiological, health-related outcomes such as improvements in cardiorespiratory fitness and cardiovascular disease risk factors compared with traditional moderate-intensity exercise in patient with heart disease ${ }^{80-83}$ and cancer. ${ }^{84}$ Also, in terms of the tumour microenvironment, higher-intensity exercise seems to be required to induce a sufficient increase in NK cell infiltration to kill tumour cells (up to $60 \%$ in animal models ${ }^{23}$ with other potential anticancer properties (eg, modulating inflammation, oxidative stress, lactate and insulin resistance).${ }^{85} 86$ Finally, HIIT has been tested in several types of cancer patients and is not only feasible and safe,${ }^{87-91}$ but also provides better physiological and psychosocial outcomes, ${ }^{8492}$ exercise adherence ${ }^{849293}$ and cost-effectiveness. ${ }^{93}$

Our model (figure 1) proposes that a sufficiently delivered dose and intensity of exercise, indicated by improvement in cardiorespiratory fitness, may benefit men with PCa on AS through three distinct pathways: (1) In the 'biological pathway', exercise may have a direct effect on suppressing tumour growth, thereby, delaying or preventing the need for radical treatments in AS patients. Scientific evidence shows that exercise can modulate cancer-related circulating markers in various mechanisms ${ }^{94-97}$ such as immune functions (eg, NK cell mobilisation and infiltration into cancer cells), ${ }^{98-101}$ inflammation (eg, systemic concentrations of IL-6, TNF- $\alpha$ and CRP) ${ }^{102}$ and metabolism (eg, insulin and IGF-axis), ${ }^{19} 22$ which creates an antitumour microenvironment resulting in a delay or even reversing of tumour progression. ${ }^{97}$ (2) In the 'psychological pathway', exercise may reduce the fear of cancer progression through several psychological mechanisms such as providing a distraction and a sense of control, reducing anxiety/cancer worry and intrusive thoughts and providing an alternative explanation for everyday symptoms such as mild pain, fatigue and soreness that may be misinterpreted as signs of cancer progression. ${ }^{12} 13$ These psychological benefits may also improve quality of life and prevent these men from requesting radical treatments as a way of managing their anxiety and fear of cancer progression. (3) In the 'functional pathway', exercise can improve overall physical function, aerobic fitness, muscular strength, body composition and activities of daily living. Improvements in these factors may help reduce the impact and complications of radical treatments and improve long-term prognosis. If PCa patients on AS feel they are better prepared physically for radical treatments and that they will experience fewer side effects and complications, they may also reduce their fear of cancer progression.

There are several strengths of the ERASE trial. It will be one of the few exercise oncology studies in the AS setting to evaluate the isolated effects of exercise. Previous exercise studies in PCa patients have focused on patients during or after treatments, particularly androgen deprivation therapy. Findings from patients receiving or recovering from active treatments are unable to answer important questions of $\mathrm{PCa}$ patients on AS related to disease 
progression, fear of cancer progression and preparation for treatments. Second, to the best of our knowledge, our study will be the first clinical trial to evaluate HIIT aerobic exercise in AS patients. Recent exercise oncology studies have tested different exercise intensities (ie, moderate-to-vigorous intensity continuous aerobic exercise), ${ }^{6768}$ modalities (ie, aerobic and resistance combined), ${ }^{68}$ or settings (ie, supervised plus home-based), ${ }^{67}$ which may have different impacts on physiological and psychosocial responses as well as on feasibility outcomes. Based on up-todate evidence in exercise oncology, HIIT aerobic exercise can be safe and feasible in this population and may be a superior modality for improving tumour suppression and clinical outcomes. Third, ERASE will be the first exercise randomised controlled trial to examine fear of cancer progression as an outcome. This psychological construct has emerged as a major unmet need in the psychological management of cancer patients and, especially in the AS setting, it has been proposed that it may actually influence treatment decisions. ERASE will provide evidence whether patient-reported fear of cancer progression can be modulated by HIIT exercise. Fourth, ERASE will explore the effects of exercise on potential cancer-related outcomes. Recent epidemiological and preclinical evidence suggests a positive association between exercise and PCa survival and a potential biological link between exercise and tumour growth and metastasis ${ }^{97}$; however, this question has not been studied in a clinical setting. We will assess cancer-related biomarkers as well as immune, metabolic, inflammatory and sex hormones to identify exploratory evidence of the potential antitumour mechanisms of exercise. Also, we will follow-up with participant medical records to document progression to radical treatment up to 1 year. Limitations of the ERASE trial include the lack of ability to blind participants and interventionists to group allocation, the self-selection sampling bias, the relatively short periods of intervention and follow-up and insufficient power to examine clinical outcomes.

In summary, the ERASE trial is expected to provide preliminary evidence that exercise improves physical fitness, manages fear of cancer progression and suppresses tumour-progression related biomarkers in men with PCa receiving AS. This study will advance knowledge and inform larger phase II and III trials designed to determine the effects of exercise on clinically important outcomes in this patient population, including progression to radical treatments, short-term complications after radical treatment and long-term outcomes related to recurrence, metastasis and overall survival. If exercise can be shown to suppress tumour progression, reduce fear of cancer progression and help men with PCa remain on AS longer, it would represent a critical advancement in the treatment and care of PCa patients receiving AS.

\section{ETHICS AND DISSEMINATION}

The ultimate purpose of the ERASE trial is to change AS clinical practice by disseminating our findings to clinicians and cancer organisations involved in the care of patients. We will disseminate our findings to these groups including presentations at annual conferences and publications in scientific journals that have a clinical focus. Also, we will directly educate PCa patients and their families about our research findings by actively using media, educational materials, and outreach to hospitals and patient advocacy groups as it is crucial to educate AS patients on the potential benefits of exercise on their physiological and psychological health and encourage them to eventually participate in exercise.

Contributors DK, AF, NB, CF and KC contributed to the design and development of the study and the final review of the manuscript. DK and KC wrote the manuscript with input from all authors.

Funding This study is supported by the Canadian Institutes of Health Research (Grant Number: RN335581 - 389507) and Prostate Cancer Canada (Grant Number: D2017-1820). Dong-Woo Kang is supported by the Alberta Innovates Graduate Studentship. Kerry S. Courneya is supported by the Canada Research Chairs Program.

Competing interests None declared.

Patient consent for publication Not required.

Ethics approval The study has received full ethical approval from the HealthResearch Ethics Board of Alberta - Cancer Committee (Protocol Number:HREBA.CC-17-0248).

Provenance and peer review Not commissioned; externally peer reviewed.

Open access This is an open access article distributed in accordance with the Creative Commons Attribution Non Commercial (CC BY-NC 4.0) license, which permits others to distribute, remix, adapt, build upon this work non-commercially, and license their derivative works on different terms, provided the original work is properly cited, appropriate credit is given, any changes made indicated, and the use is non-commercial. See: http://creativecommons.org/licenses/by-nc/4.0/.

\section{REFERENCES}

1. Heidenreich A, Bellmunt J, Bolla M, et al. EAU guidelines on prostate cancer. Part 1: screening, diagnosis, and treatment of clinically localised disease. Eur Urol 2011;59:61-71.

2. Talcott JA, Rieker P, Clark JA, et al. Patient-reported symptoms after primary therapy for early prostate cancer: results of a prospective cohort study. J Clin Oncol 1998;16:275-83.

3. Donovan JL, Hamdy FC, Lane JA, et al. Patient-reported outcomes after monitoring, surgery, or radiotherapy for prostate cancer. $N$ Engl J Med Overseas Ed 2016;375:1425-37.

4. Chen RC, Basak R, Meyer AM, et al. Association between choice of radical prostatectomy, external beam radiotherapy, brachytherapy, or active surveillance and patient-reported quality of life among men with localized prostate cancer. JAMA 2017;317:1141-50.

5. Cooperberg MR, Carroll PR, Klotz L. Active surveillance for prostate cancer: progress and promise. J Clin Oncol 2011;29:3669-76.

6. Hamdy FC, Donovan JL, Lane JA, et al. 10-Year outcomes after monitoring, surgery, or radiotherapy for localized prostate cancer. $N$ Engl J Med Overseas Ed 2016;375:1415-24.

7. Dall'Era MA. The economics of active surveillance for prostate cancer. Curr Opin Urol 2013;23:278-82.

8. Keegan KA, Dall'Era MA, Durbin-Johnson B, et al. Active surveillance for prostate cancer compared with immediate treatment: an economic analysis. Cancer 2012;118:3512-8.

9. Lao C, Edlin R, Rouse P, et al. The cost-effectiveness of active surveillance compared to watchful waiting and radical prostatectomy for low risk localised prostate cancer. BMC Cancer 2017; 17:529.

10. Dragomir A, Cury FL, Aprikian AG. Active surveillance for low-risk prostate cancer compared with immediate treatment: a Canadian cost comparison. CMAJ Open 2014;2:E60-8.

11. Marzouk K, Assel M, Ehdaie B, et al. Long-term cancer specific anxiety in men undergoing active surveillance of prostate cancer: findings from a large prospective cohort. J Urol 2018;200:1250-5. 
12. Wallace M. Uncertainty and quality of life of older men who undergo watchful waiting for prostate cancer. Oncol Nurs Forum 2003;30:303-9.

13. Parker PA, Davis JW, Latini DM, et al. Relationship between illness uncertainty, anxiety, fear of progression and quality of life in men with favourable-risk prostate cancer undergoing active surveillance. BJU Int 2016;117:469-77.

14. Kinsella N, Stattin P, Cahill D, et al. Factors influencing men's choice of and adherence to active surveillance for low-risk prostate cancer: a mixed-method systematic review. Eur Urol 2018;74:261-80.

15. Matthew AG, Raz O, Currie KL, et al. Psychological distress and lifestyle disruption in low-risk prostate cancer patients: Comparison between active surveillance and radical prostatectomy. J Psychosoc Oncol 2018;36:159-74.

16. Latini DM, Hart SL, Knight SJ, et al. The relationship between anxiety and time to treatment for patients with prostate cancer on surveillance. J Urol 2007;178:826-32.

17. van den Bergh $\mathrm{RC}$, Korfage IJ, Bangma $\mathrm{CH}$. Psychological aspects of active surveillance. Curr Opin Urol 2012;22:237-42.

18. Hvid $\mathrm{T}$, Lindegaard $\mathrm{B}$, Winding $\mathrm{K}$, et al. Effect of a 2 -year homebased endurance training intervention on physiological function and PSA doubling time in prostate cancer patients. Cancer Causes Control 2016;27:165-74.

19. Leung PS, Aronson WJ, Ngo TH, et al. Exercise alters the IGF axis in vivo and increases p53 protein in prostate tumor cells in vitro. $J$ Appl Physiol 2004;96:450-4.

20. Jones LW, Antonelli J, Masko EM, et al. Exercise modulation of the host-tumor interaction in an orthotopic model of murine prostate cancer. J Appl Physiol 2012;113:263-72.

21. McCullough DJ, Nguyen LM, Siemann DW, et al. Effects of exercise training on tumor hypoxia and vascular function in the rodent preclinical orthotopic prostate cancer model. J Appl Physiol 2013;115:1846-54.

22. Rundqvist $H$, Augsten $M$, Strömberg $A$, et al. Effect of acute exercise on prostate cancer cell growth. PLoS One 2013;8:e67579.

23. Pedersen $\mathrm{L}$, Idorn $\mathrm{M}$, Olofsson $\mathrm{GH}$, et al. Voluntary running suppresses tumor growth through epinephrine- and il-6-dependent NK cell mobilization and redistribution. Cell Metab 2016;23:554-62.

24. Jones LW, Hornsby WE, Freedland SJ, et al. Effects of nonlinear aerobic training on erectile dysfunction and cardiovascular function following radical prostatectomy for clinically localized prostate cancer. Eur Urol 2014;65:852-5.

25. Cormie P, Newton RU, Spry N, et al. Safety and efficacy of resistance exercise in prostate cancer patients with bone metastases. Prostate Cancer Prostatic Dis 2013;16:328-35.

26. Cormie P, Galvão DA, Spry N, et al. Can supervised exercise prevent treatment toxicity in patients with prostate cancer initiating androgen-deprivation therapy: a randomised controlled trial. BJU Int 2015;115:256-66.

27. Segal RJ, Reid RD, Courneya KS, et al. Randomized controlled trial of resistance or aerobic exercise in men receiving radiation therapy for prostate cancer. J Clin Oncol 2009;27:344-51.

28. Galvão DA, Taaffe DR, Spry N, et al. Combined resistance and aerobic exercise program reverses muscle loss in men undergoing androgen suppression therapy for prostate cancer without bone metastases: a randomized controlled trial. J Clin Oncol 2010;28:340-7.

29. Park SW, Kim TN, Nam JK, et al. Recovery of overall exercise ability, quality of life, and continence after 12-week combined exercise intervention in elderly patients who underwent radica prostatectomy: a randomized controlled study. Urology 2012;80:299-306.

30. Segal RJ, Reid RD, Courneya KS, et al. Resistance exercise in men receiving androgen deprivation therapy for prostate cancer. J Clin Oncol 2003;21:1653-9.

31. Farris MS, Kopciuk KA, Courneya KS, et al. Identification and prediction of health-related quality of life trajectories after a prostate cancer diagnosis. Int J Cancer 2017;140:1517-27.

32. Silver JK, Baima J. Cancer prehabilitation: an opportunity to decrease treatment-related morbidity, increase cancer treatment options, and improve physical and psychological health outcomes. Am J Phys Med Rehabil 2013;92:715-27.

33. National Comprehansive Cancer Network (NCCN). NCCN Clinical Practice Guidelines in Oncology (NCCN Guidelines $\left.{ }^{\circledR}\right)$ Prostate Cancer. 2017.

34. Warburton DE, Jamnik VK, Bredin SS, et al. The 2011 Physical Activity Readiness Questionnaire for Everyone (PAR-Q+) and the Electronic Physical Activity Readiness Medical Examination (ePARmed-X+). Heal Fit J Canada 2011;4.

35. Godin G, Shephard R. The godin-shephard leisure-time physical activity questionnaire. Med Sci Sports Exerc 1997;29:36-8.
36. Alberta Cancer Exercise (ACE) Program. https://www.albertacance rexercise.com/.

37. McINNIS K, Balady GJ. Comparison of suhmaximal exercise responses using the Bruce vs modified Bruce protocols. Medicine \& Science in Sports \& Exercise 1994;26:103-7.

38. Burdon JG, Juniper EF, Killian KJ, et al. The perception of breathlessness in asthma. Am Rev Respir Dis 1982;126:825-8.

39. Simard S, Savard J. Fear of Cancer Recurrence Inventory: development and initial validation of a multidimensional measure of fear of cancer recurrence. Support Care Cancer 2009;17:241-51.

40. Custers JA, van den Berg SW, van Laarhoven HW, et al. The cancer worry scale: detecting fear of recurrence in breast cancer survivors. Cancer Nurs 2014;37:E44-50.

41. Aaronson NK, Ahmedzai S, Bergman B, et al. The European Organization for Research and Treatment of Cancer QLQ-C30: a quality-of-life instrument for use in international clinical trials in oncology. J Natl Cancer Inst 1993;85:365-76.

42. Spielberger CD. State-Trait anxiety inventory: Wiley Online Library. 2010.

43. Roth AJ, Rosenfeld B, Kornblith $A B$, et al. The memorial anxiety scale for prostate cancer: validation of a new scale to measure anxiety in men with with prostate cancer. Cancer 2003;97:2910-8.

44. Lewinsohn PM, Seeley JR, Roberts RE, et al. Center for Epidemiologic Studies Depression Scale (CES-D) as a screening instrument for depression among community-residing older adults. Psychol Aging 1997;12:277-87.

45. Yellen SB, Cella DF, Webster K, et al. Measuring fatigue and other anemia-related symptoms with the Functional Assessment of Cancer Therapy (FACT) measurement system. J Pain Symptom Manage 1997;13:63-74.

46. Cohen S, Kamarck T, Mermelstein R. Perceived stress scale. Measuring stress: a guide for health and social scientists, 1994.

47. Rosenberg M. Rosenberg self-esteem scale (RSE). Acceptance and commitment therapy Measures package. 1965;61:52.

48. Ajzen I. The theory of planned behavior. Organ Behav Hum Decis Process 1991;50:179-211.

49. Rikli RE, Jones CJ. Senior fitness test manual: Human Kinetics. 2013.

50. American College of Sports Medicine. ACSM's guidelines for exercise testing and prescription: Lippincott Williams \& Wilkins, 2013.

51. Borm GF, Fransen J, Lemmens WA. A simple sample size formula for analysis of covariance in randomized clinical trials. J Clin Epidemiol 2007;60:1234-8.

52. Courneya KS, Mackey JR, Bell GJ, et al. Randomized controlled trial of exercise training in postmenopausal breast cancer survivors: cardiopulmonary and quality of life outcomes. J Clin Oncol 2003;21:1660-8.

53. Courneya KS, Segal RJ, Mackey JR, et al. Effects of aerobic and resistance exercise in breast cancer patients receiving adjuvant chemotherapy: a multicenter randomized controlled trial. J Clin Oncol 2007:25:4396-404.

54. Courneya KS, Sellar CM, Stevinson C, et al. Randomized controlled trial of the effects of aerobic exercise on physical functioning and quality of life in lymphoma patients. J Clin Oncol 2009;27:4605-12.

55. Norris MK, Bell GJ, North S, et al. Effects of resistance training frequency on physical functioning and quality of life in prostate cancer survivors: a pilot randomized controlled trial. Prostate Cancer Prostatic Dis 2015;18:281-7.

56. Harris PA, Taylor R, Thielke R, et al. Research electronic data capture (REDCap)--a metadata-driven methodology and workflow process for providing translational research informatics support. $J$ Biomed Inform 2009;42:377-81.

57. Royston P. Multiple Imputation of Missing Values. Stata J 2004;4:227-41.

58. Ornish D, Weidner G, Fair WR, et al. Intensive lifestyle changes may affect the progression of prostate cancer. J Urol 2005;174:1065-70.

59. Ornish DM, Lee KL, Fair WR, et al. Dietary trial in prostate cancer: early experience and implications for clinical trial design. Urology 2001:57:200-1.

60. Daubenmier JJ, Weidner G, Marlin R, et al. Lifestyle and healthrelated quality of life of men with prostate cancer managed with active surveillance. Urology 2006;67:125-30.

61. Ornish D, Lin J, Daubenmier J, et al. Increased telomerase activity and comprehensive lifestyle changes: a pilot study. Lancet Oncol 2008;9:1048-57.

62. Frattaroli J, Weidner G, Dnistrian AM, et al. Clinical events in prostate cancer lifestyle trial: results from two years of follow-up. Urology 2008;72:1319-23.

63. Eriksen AK, Hansen RD, Borre M, et al. A lifestyle intervention among elderly men on active surveillance for non-aggressive 
prostate cancer: a randomised feasibility study with whole-grain rye and exercise. Trials 2017;18:20.

64. Kenfield SA, Chang ST, Chan JM. Diet and lifestyle interventions in active surveillance patients with favorable-risk prostate cancer. Cur Treat Options Oncol 2007;8:173-96.

65. Burton AJ, Martin RM, Donovan JL, et al. Associations of lifestyle factors and anthropometric measures with repeat PSA levels during active surveillance/monitoring. Cancer Epidemiol Biomarkers Prev 2012;21:1877-85.

66. Vandersluis AD, Guy DE, Klotz LH, et al. The role of lifestyle characteristics on prostate cancer progression in two active surveillance cohorts. Prostate Cancer Prostatic Dis 2016;19:305-10.

67. Bourke L, Stevenson R, Turner R, et al. Exercise training as a novel primary treatment for localised prostate cancer: a multi-site randomised controlled phase II study. Sci Rep 2018;8:8374.

68. Galvão DA, Hayne D, Frydenberg M, et al. Can exercise delay transition to active therapy in men with low-grade prostate cancer? A multicentre randomised controlled trial. BMJ Open 2018;8:e022331.

69. Lakoski SG, Willis BL, Barlow CE, et al. Midlife cardiorespiratory fitness, incident cancer, and survival after cancer in men: the cooper center longitudinal study. JAMA Oncol 2015;1:231-7.

70. Oliveria SA, Kohl HW, Trichopoulos D, et al. The association between cardiorespiratory fitness and prostate cancer. Med Sci Sports Exerc 1996;28:97-104.

71. Van Hemelrijck M, Garmo H, Holmberg L, et al. Absolute and relative risk of cardiovascular disease in men with prostate cancer: results from the population-based PCBaSe Sweden. $J$ Clin Oncol 2010;28:3448-56.

72. Gupta D, Lee Chuy K, Yang JC, et al. Cardiovascular and metabolic effects of androgen-deprivation therapy for prostate cancer. J Oncol Pract 2018;14:580-7.

73. Matthes KL, Pestoni G, Korol D, et al. The risk of prostate cancer mortality and cardiovascular mortality of nonmetastatic prostate cancer patients: A population-based retrospective cohort study. Urol Oncol 2018:36:309.e15-23.

74. Myers J, Prakash M, Froelicher V, et al. Exercise capacity and mortality among men referred for exercise testing. $N$ Engl J Med 2002;346:793-801.

75. Kodama S, Saito K, Tanaka S, et al. Cardiorespiratory fitness as a quantitative predictor of all-cause mortality and cardiovascular events in healthy men and women: a meta-analysis. JAMA 2009;301:2024-35.

76. Scott JM, Zabor EC, Schwitzer E, et al. Efficacy of exercise therapy on cardiorespiratory fitness in patients with cancer: a systematic review and meta-analysis. J Clin Oncol 2018;36:2297-305.

77. Helgerud J, Høydal K, Wang E, et al. Aerobic high-intensity intervals improve VO2max more than moderate training. Med Sci Sports Exerc 2007;39:665-71.

78. Ramos JS, Dalleck LC, Tjonna AE, et al. The impact of highintensity interval training versus moderate-intensity continuous training on vascular function: a systematic review and metaanalysis. Sports Med 2015;45:679-92.

79. Gibala MJ, Little JP, Macdonald MJ, et al. Physiological adaptations to low-volume, high-intensity interval training in health and disease. J Physiol 2012;590:1077-84.

80. Cardozo GG, Oliveira RB, Farinatti PT. Effects of high intensity interval versus moderate continuous training on markers of ventilatory and cardiac efficiency in coronary heart disease patients. ScientificWorldJournal 2015;2015:1-8.

81. Weston KS, Wisløff U, Coombes JS. High-intensity interval training in patients with lifestyle-induced cardiometabolic disease: a systematic review and meta-analysis. Br J Sports Med 2014:48:1227-34.

82. Warburton DE, McKenzie DC, Haykowsky MJ, et al. Effectiveness of high-intensity interval training for the rehabilitation of patients with coronary artery disease. Am J Cardiol 2005;95:1080-4.
83. Moholdt TT, Amundsen BH, Rustad LA, et al. Aerobic interval training versus continuous moderate exercise after coronary artery bypass surgery: a randomized study of cardiovascular effects and quality of life. Am Heart J 2009;158:1031-7.

84. Toohey K, Pumpa K, McKune A, et al. High-intensity exercise interventions in cancer survivors: a systematic review exploring the impact on health outcomes. J Cancer Res Clin Oncol 2018;144:1-12

85. Papadopoulos E, Santa Mina D. Can we HIIT cancer if we attack inflammation? Cancer Causes Control 2018;29:7-11.

86. Hofmann P. Cancer and exercise: warburg hypothesis, tumour metabolism and high-intensity anaerobic exercise. Sports 2018;6:E10.

87. Banerjee S, Manley K, Shaw B, et al. Vigorous intensity aerobic interval exercise in bladder cancer patients prior to radical cystectomy: a feasibility randomised controlled trial. Support Care Cancer 2018;26:1515-23.

88. Licker M, Karenovics W, Diaper J, et al. Short-term preoperative high-intensity interval training in patients awaiting lung cancer surgery: a randomized controlled trial. J Thorac Oncol 2017:12:323-33.

89. Schmitt J, Lindner N, Reuss-Borst M, et al. A 3-week multimodal intervention involving high-intensity interval training in female cancer survivors: a randomized controlled trial. Physiol Rep 2016;4:e12693.

90. Schulz SVW, Laszlo R, Otto S, et al. Feasibility and effects of a combined adjuvant high-intensity interval/strength training in breast cancer patients: a single-center pilot study. Disabil Rehabil 2018;40:1501-8

91. Toohey K, Pumpa KL, Arnolda L, et al. A pilot study examining the effects of low-volume high-intensity interval training and continuous low to moderate intensity training on quality of life, functional capacity and cardiovascular risk factors in cancer survivors. PeerJ 2016;4:e2613.

92. Adams SC, DeLorey DS, Davenport MH, et al. Effects of highintensity aerobic interval training on cardiovascular disease risk in testicular cancer survivors: A phase 2 randomized controlled trial. Cancer 2017;123:4057-65.

93. Kampshoff CS, van Dongen JM, van Mechelen W, et al. Longterm effectiveness and cost-effectiveness of high versus low-tomoderate intensity resistance and endurance exercise interventions among cancer survivors. J Cancer Surviv 2018;12:417-29.

94. Hayes BD, Brady L, Pollak M, et al. Exercise and prostate cancer: evidence and proposed mechanisms for disease modification. Cancer Epidemiol Biomarkers Prev 2016;25:1281-8.

95. Ruiz-Casado A, Martín-Ruiz A, Pérez LM, et al. Exercise and the hallmarks of cancer. Trends Cancer 2017;3:423-41.

96. Hojman P, Gehl J, Christensen JF, et al. Molecular mechanisms linking exercise to cancer prevention and treatment. Cell Metab 2018;27:10-21.

97. Ashcraft KA, Peace RM, Betof AS, et al. Efficacy and mechanisms of aerobic exercise on cancer initiation, progression, and metastasis: a critical systematic review of In Vivo preclinical data. Cancer Res 2016;76:4032-50.

98. Bigley AB, Simpson RJ. NK cells and exercise: implications for cancer immunotherapy and survivorship. Discov Med 2015;19:433-45.

99. Idorn M, Hojman P. Exercise-dependent regulation of NK cells in cancer protection. Trends Mol Med 2016;22:565-77.

100. Idorn M, Thor Straten P. Exercise: A new role for an old tool. Mol Cell Oncol 2016;3:e1163005.

101. Simpson RJ, Bigley AB, Agha N, et al. Mobilizing immune cells with exercise for cancer immunotherapy. Exerc Sport Sci Rev 2017:45:163-72.

102. Gleeson M, Bishop NC, Stensel DJ, et al. The anti-inflammatory effects of exercise: mechanisms and implications for the prevention and treatment of disease. Nat Rev Immunol 2011;11:607-15. 